\title{
Renormalization aspects of $\mathcal{N}=1$ Super Yang-Mills theory in the Wess-Zumino gauge
}

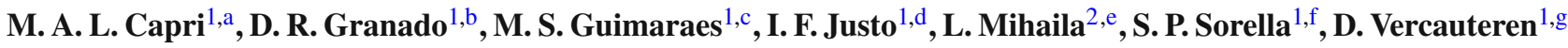 \\ ${ }^{1}$ Departamento de Física Teórica, Instituto de Física, UERJ-Universidade do Estado do Rio de Janeiro, Rua São Francisco Xavier 524, Maracanã, \\ Rio de Janeiro 20550-013, Brazil \\ ${ }^{2}$ Institut für Theoretische Teilchenphysik, Karlsruhe Institute of Technology (KIT), 76128 Karlsruhe, Germany
}

Received: 29 January 2014 / Accepted: 1 April 2014 / Published online: 23 April 2014

(C) The Author(s) 2014. This article is published with open access at Springerlink.com

\begin{abstract}
The renormalization of $\mathcal{N}=1$ Super YangMills theory is analyzed in the Wess-Zumino gauge, employing the Landau condition. An all-orders proof of the renormalizability of the theory is given by means of the Algebraic Renormalization procedure. Only three renormalization constants are needed, which can be identified with the coupling constant, gauge field, and gluino renormalization. The nonrenormalization theorem of the gluon-ghost-antighost vertex in the Landau gauge is shown to remain valid in $\mathcal{N}=1$ Super Yang-Mills. Moreover, due to the non-linear realization of the supersymmetry in the Wess-Zumino gauge, the renormalization factor of the gauge field turns out to be different from that of the gluino. These features are explicitly checked through a three-loop calculation.
\end{abstract}

\section{Introduction}

Supersymmetric $\mathcal{N}=1$ gauge theories exhibit remarkable features, both at perturbative and non-perturbative level; see, for instance, [1] and references therein.

For what concerns the ultraviolet behavior, the symmetry between bosons and fermions gives rise to milder divergences in the ultraviolet regime, a property which is at the origin of a set of non-renormalization theorems; see [2].

Work supported by FAPERJ, Fundação de Amparo à Pesquisa do Estado do Rio de Janeiro, under the program Cientista do Nosso Estado, E-26/101.578/2010.

\footnotetext{
a e-mail: caprimarcio@gmail.com

b e-mail: diegorochagrana@uerj.br

c e-mail: msguimaraes@uerj.br

d e-mail: igorfjusto@gmail.com

e e-mail: luminita-nicoletta.mihaila@kit.edu

fe-mail: sorella@uerj.br

g e-mail: vercauteren.uerj@gmail.com
}

In this work we discuss some features of the renormalization of $\mathcal{N}=1$ Super Yang-Mills theories in Euclidean space-time in the Wess-Zumino gauge, in which the number of field components is minimum. Employing the algebraic renormalization method [3], we are able to show, to all orders of perturbation theory, that, in the Landau gauge, only three independent renormalization factors, $\left(Z_{g}, Z_{A}, Z_{\lambda}\right)$, are needed to renormalize the theory, which can be identified with the coupling constant, gauge field and gluino renormalization. The renormalization factors of all other fields, i.e. the Lagrange multiplier implementing the Landau gauge condition, the Faddeev-Popov ghosts, the external BRST sources, the global SUSY ghosts, etc., can be expressed as suitable combinations of $\left(Z_{g}, Z_{A}, Z_{\lambda}\right)$. In particular, the nonrenormalization theorem of the gluon-ghost-antighost vertex in the Landau gauge, i.e. $Z_{g} Z_{A}^{1 / 2} Z_{c}^{1 / 2} Z_{\bar{c}}^{1 / 2}=1$, still holds in $\mathcal{N}=1$ Super Yang-Mills theories, due to the existence of the so-called ghost Ward identity; see (22). Moreover, due to the non-linear realization of supersymmetry in the WessZumino gauge, it turns out that the renormalization factor $Z_{A}$ of the gauge field is different from the renormalization factor $Z_{\lambda}$ of the gluino, a property which we shall check through a three-loop calculation and which was already observed at one-loop level in the Feynman gauge [4]. To some extent, the present work can be seen as a continuation of the work done by [5-10,12-14] in which the renormalization of supersymmetric gauge theories in the Wess-Zumino gauge was faced by using BRST cohomology tools.

The paper is organized as follows. In Sect. 2 we discuss the BRST quantization of the theory in the Wess-Zumino gauge. In Sect. 3 we derive the large set of Ward identities fulfilled by the quantized action. Further, we determine the most general invariant counterterm and find the renormalization factors of all fields, coupling constant, and external BRST sources. Section 4 is devoted to the explicit evaluation of the gauge field and gluino renormalization factors 
$\left(Z_{A}, Z_{\lambda}\right)$ as well as of the non-renormalization of the gluonghost-antighost vertex, $Z_{g} Z_{A}^{1 / 2} Z_{c}^{1 / 2} Z_{\bar{c}}^{1 / 2}=1$. In Sect. 5 we collect our conclusions. Finally, Appendix A is devoted to notations and conventions.

\section{Quantization of $\mathcal{N}=1$ Super Yang-Mills in the Wess-Zumino gauge}

As already mentioned, the advantage of the Wess-Zumino gauge is that the number of field components is minimum. There is, however, a drawback: the supersymmetry algebra is realized in a non-linear way. More precisely, the algebra of the generators of the supersymmetry $\delta_{\alpha}, \alpha=1,2,3,4$, does not close on translations. Instead, we have

$\left\{\delta_{\alpha}, \delta_{\beta}\right\}=\left(\gamma_{\mu}\right)_{\alpha \beta} \partial_{\mu}+$ (gauge transf.) + (field eqs.).

As shown in [5-10], the most powerful and efficient way to deal with the algebra (1) is constructing a generalized BRST operator $Q$ which collects both gauge and SUSY field transformations, namely

$Q=s+\epsilon^{\alpha} \delta_{\alpha}$,

where $s$ is the usual BRST operator for gauge transformations and $\epsilon^{\alpha}$ is a constant Majorana spinor parameter carrying ghost number 1 . To some extent, $\epsilon^{\alpha}$ represents the ghost corresponding to the SUSY generators. The operator $Q$ enjoys the following important property:

$Q^{2}=\epsilon^{\alpha}\left(\gamma_{\mu}\right)_{\alpha \beta} \bar{\epsilon}^{\beta} \partial_{\mu}$,

which enables us to quantize the theory by following the BRST gauge-fixing procedure in a manifestly supersymmetric invariant way.

Let us proceed by showing how this construction applies to $\mathcal{N}=1$ Super Yang-Mills theory, whose classical action in Euclidean space ${ }^{1}$ reads

$S_{\mathrm{SYM}}=\int \mathrm{d}^{4} x\left[\frac{1}{4} F_{\mu \nu}^{a} F_{\mu \nu}^{a}+\frac{1}{2} \bar{\lambda}^{a \alpha}\left(\gamma_{\mu}\right)_{\alpha \beta} D_{\mu}^{a b} \lambda^{b \beta}+\frac{1}{2} \mathfrak{D}^{a} \mathfrak{D}^{a}\right]$,

where $D_{\mu}^{a b}=\left(\delta^{a b} \partial_{\mu}+g f^{a c b} A_{\mu}^{c}\right)$ is the covariant derivative in the adjoint representation of the gauge group $S U(N), \lambda^{a \alpha}$ is a Majorana spinor, $\mathfrak{D}^{a}$ is an auxiliary field and

$F_{\mu \nu}^{a}=\partial_{\mu} A_{\nu}^{a}-\partial_{\nu} A_{\mu}^{a}+\mathrm{gf}^{a b c} A_{\mu}^{b} A_{\nu}^{c}$.

\footnotetext{
$\overline{1}$ Although we are employing here the Euclidean formulation of the theory, it is worth to point that, as far perturbation theory is concerned, the Minkowski space-time can be related to the Euclidean one through a Wick rotation. In the present paper we shall limit ourselves to perturbation theory.
}

The transformation of each field under the generalised BRST operator $Q$ reads

$$
\begin{aligned}
& Q A_{\mu}^{a}=-D_{\mu}^{a b} c^{b}+\bar{\epsilon}^{\alpha}\left(\gamma_{\mu}\right)_{\alpha \beta} \lambda^{a \beta}, \\
& Q \lambda^{a \alpha}=\mathrm{gf}^{a b c} c^{b} \lambda^{c \alpha}-\frac{1}{2}\left(\sigma_{\mu \nu}\right)^{\alpha \beta} \epsilon_{\beta} F_{\mu \nu}^{a}+\left(\gamma_{5}\right)^{\alpha \beta} \epsilon_{\beta} \mathfrak{D}^{a}, \\
& Q \mathfrak{D}^{a}=\mathrm{gf}^{a b c} c^{b} \mathfrak{D}^{c}+\bar{\epsilon}^{\alpha}\left(\gamma_{\mu}\right)_{\alpha \beta}\left(\gamma_{5}\right)^{\beta \eta} D_{\mu}^{a b} \lambda_{\eta}^{b}, \\
& Q c^{a}=\frac{1}{2} \mathrm{gf}^{a b c} c^{b} c^{c}-\bar{\epsilon}^{\alpha}\left(\gamma_{\mu}\right)_{\alpha \beta} \epsilon^{\beta} A_{\mu}^{a}, \\
& Q \bar{c}^{a}=b^{a} \\
& Q b^{a}=\nabla \bar{c}^{a}, \\
& Q^{2}=\nabla,
\end{aligned}
$$

where we have introduced the translation operator

$\nabla:=\bar{\epsilon}^{\alpha}\left(\gamma_{\mu}\right)_{\alpha \beta} \epsilon^{\beta} \partial_{\mu}$.

The fields $\left(\bar{c}^{a}, c^{a}\right)$ stand for the Faddeev-Popov ghosts, while $b^{a}$ is the Lagrange multiplier needed to implement the Landau gauge fixing, $\partial_{\mu} A_{\mu}^{a}=0$. It is easy to check that the action (4) is left invariant by the transformations (6), i.e.

$Q S_{\mathrm{SYM}}=0$.

In order to quantize the theory, we need to introduce the gauge-fixing term. This task can be accomplished by following the BRST construction, amounting to introduce the gauge condition in a $Q$-exact way. One should notice that, owing to property (3), the generalised BRST operator $Q$ is in fact nilpotent when acting on space-time integrated polynomials in the fields and their derivatives. Adopting the Landau gauge, $\partial_{\mu} A_{\mu}^{a}=0$, for the gauge-fixing term we write

$S_{\mathrm{gf}}=Q \int \mathrm{d}^{4} x\left(\bar{c}^{a} \partial_{\mu} A_{\mu}^{a}\right)$,

so that, according to (6)

$S_{\mathrm{gf}}=\int \mathrm{d}^{4} x\left[\bar{c}^{a} \partial_{\mu} D_{\mu}^{a b} c^{b}+b^{a} \partial_{\mu} A_{\mu}^{a}-\bar{c}^{a} \bar{\epsilon}^{\alpha}\left(\gamma_{\mu}\right)_{\alpha \beta} \partial_{\mu} \lambda^{a \beta}\right]$.

Therefore, the Super Yang-Mills action in the Wess-Zumino and Landau gauge can be written as

$$
\begin{aligned}
S= & S_{\mathrm{SYM}}+S_{\mathrm{gf}} \\
= & \int \mathrm{d}^{4} x\left\{\frac{1}{4} F_{\mu \nu}^{a} F_{\mu \nu}^{a}+\frac{1}{2} \bar{\lambda}^{a \alpha}\left(\gamma_{\mu}\right)_{\alpha \beta} D_{\mu}^{a b} \lambda^{b \beta}+\frac{1}{2} \mathfrak{D}^{2}\right. \\
& \left.+b^{a} \partial_{\mu} A_{\mu}^{a}+\bar{c}^{a}\left[\partial_{\mu} D_{\mu}^{a b} c^{b}-\bar{\epsilon}^{\alpha}\left(\gamma_{\mu}\right)_{\alpha \beta} \partial_{\mu} \lambda^{a \beta}\right]\right\} .
\end{aligned}
$$

From (6), (8), and (9), it follows immediately that

$Q S=0$,

meaning that the gauge-fixing procedure has been done in a BRST invariant way. Moreover, recalling that the generalized 
operator $Q$ collects both gauge and supersymmetry transformations, one realizes that the expression (10) is the supersymmetric generalization of the Landau gauge, as can be inferred from the presence of the additional term $\bar{c}^{a} \bar{\epsilon}^{\alpha}\left(\gamma_{\mu}\right)_{\alpha \beta} \partial_{\mu} \lambda^{a \beta}$, which contains the supersymmetry ghost $\bar{\epsilon}^{\alpha}$ as well as the gluino field $\lambda^{a \beta}$.

Having quantized the theory, we are ready to write down the large set of Ward identities and proceed with the algebraic characterization of the most general invariant counterterm. This will be the task of the next section.

\section{Ward identities and algebraic characterization of the invariant counterterm}

In order to write down the set of Ward identities which will be employed for the algebraic analysis of the model, we need to introduce a set of external sources coupled to the non-linear transformations appearing in (6). More precisely, from (6), we need to introduce external sources coupled to $Q A_{\mu}^{a}, Q \lambda^{a \beta}, Q D^{a}$ and $Q c^{a}$. With that purpose, we introduce the following BRST doublets [3] of the sources:

$$
\begin{aligned}
& \left\{\begin{array}{c}
Q K_{\mu}^{a}=\Omega_{\mu}^{a} \\
Q \Omega_{\mu}^{a}=\nabla K_{\mu}^{a}
\end{array}, \quad\left\{\begin{array}{c}
Q L^{a}=\Lambda^{a} \\
Q \Lambda^{a}=\nabla L^{a}
\end{array}\right.\right. \\
& \left\{\begin{array}{c}
Q T^{a}=J^{a} \\
Q J^{a}=\nabla T^{a}
\end{array},\left\{\begin{array}{c}
Q Y^{a \alpha}=X^{a \alpha} \\
Q X^{a \alpha}=\nabla Y^{a \alpha}
\end{array}\right.\right.
\end{aligned}
$$

and the $Q$-exact external action

$$
\begin{aligned}
S_{\mathrm{ext}}= & Q \int \mathrm{d}^{4} x\left(-K_{\mu}^{a} A_{\mu}^{a}+L^{a} c^{a}-T^{a} \mathfrak{D}^{a}\right. \\
& \left.+Y^{a \alpha} \lambda_{\alpha}^{a}-T^{a} Y^{a \alpha}\left(\gamma_{5}\right)_{\alpha \beta} \epsilon^{\beta}\right),
\end{aligned}
$$

leading to the following complete $Q$-invariant action $\Sigma$ :

$\Sigma=S_{\mathrm{SYM}}+S_{\mathrm{gf}}+S_{\mathrm{ext}}$,

$Q \Sigma=0$.

Explicitly

$$
\begin{aligned}
\Sigma= & \int \mathrm{d}^{4} x\left\{\frac{1}{4} F_{\mu \nu}^{a} F_{\mu \nu}^{a}+\frac{1}{2} \bar{\lambda}^{a \alpha}\left(\gamma_{\mu}\right)_{\alpha \beta} D_{\mu}^{a b} \lambda^{b \beta}\right. \\
& +\frac{1}{2} \mathfrak{D}^{a} \mathfrak{D}^{a}+b^{a} \partial_{\mu} A_{\mu}^{a} \\
& +\bar{c}^{a}\left[\partial_{\mu} D_{\mu}^{a b} c^{b}-\bar{\epsilon}^{\alpha}\left(\gamma_{\mu}\right)_{\alpha \beta} \partial_{\mu} \lambda^{a \beta}\right] \\
& +T^{a}\left[\mathrm{gf}^{a b c} c^{b} \mathfrak{D}^{c}+\bar{\epsilon}^{\alpha}\left(\gamma_{\mu}\right)_{\alpha \beta}\left(\gamma_{5}\right)^{\beta \eta} D_{\mu}^{a b} \lambda_{\eta}^{b}\right] \\
& +L^{a}\left[\frac{g}{2} f^{a b c} c^{b} c^{c}-\bar{\epsilon}^{\alpha}\left(\gamma_{\mu}\right)_{\alpha \beta} \epsilon^{\beta} A_{\mu}^{a}\right] \\
& -K_{\mu}^{a}\left[D_{\mu}^{a b} c^{b}-\bar{\epsilon}^{\alpha}\left(\gamma_{\mu}\right)_{\alpha \beta} \lambda^{a \beta}\right]-\Omega_{\mu}^{a} A_{\mu}^{a} \\
& +Y^{a \alpha}\left[\mathrm{gf}^{a b c} c^{b} \lambda_{\alpha}^{c}-\frac{1}{2}\left(\sigma_{\mu \nu}\right)_{\alpha \beta} F_{\mu \nu}^{a} \epsilon^{\beta}+\left(\gamma_{5}\right)_{\alpha \beta} \epsilon^{\beta} \mathfrak{D}^{a}\right]
\end{aligned}
$$

$$
\begin{aligned}
& +\Lambda^{a} c^{a}-J^{a} \mathfrak{D}^{a}+X^{a \alpha} \lambda_{\alpha}^{a} \\
& \left.-J^{a} Y^{a \alpha}\left(\gamma_{5}\right)_{\alpha \beta} \varepsilon^{\beta}+T^{a} X^{a \alpha}\left(\gamma_{5}\right)_{\alpha \beta} \varepsilon^{\beta}\right\} \text {. }
\end{aligned}
$$

Notice that in expression (14) a term quadratic in the external sources, i.e. $T^{a} Y^{a \alpha}\left(\gamma_{5}\right)_{\alpha \beta} \epsilon^{\beta}$, has been introduced. Similar terms are present also in the analysis done by [5-10]. As we shall see, it will be needed for renormalization purposes. The external sources can be set to zero at the end, after having identified the most general counter term and all renormalization factors. The expression (17) represents the starting point for the algebraic analysis of the model, namely for the determination of the most general invariant counterterm compatible with all possible Ward identities fulfilled by $\Sigma$.

\subsection{Ward identities}

The complete action $\Sigma$ obeys a large set of Ward identities, which we display below:

- The Slavnov-Taylor identity:

$$
\mathcal{S}(\Sigma)=0
$$

where

$$
\begin{aligned}
\mathcal{S}(\Sigma)= & \int \mathrm{d}^{4} x\left\{\left(\frac{\delta \Sigma}{\delta A_{\mu}^{a}}+\Omega_{\mu}^{a}\right) \frac{\delta \Sigma}{\delta K_{\mu}^{a}}\right. \\
& +\left(\frac{\delta \Sigma}{\delta \lambda^{a \alpha}}+X^{a \alpha}\right) \frac{\delta \Sigma}{\delta Y^{a \alpha}}+\frac{\delta \Sigma}{\delta \lambda^{a \alpha}}\left(\gamma_{5}\right)_{\alpha \beta} \epsilon^{\beta} J^{a} \\
& +\left(\frac{\delta \Sigma}{\delta c^{a}}+\Lambda^{a}\right) \frac{\delta \Sigma}{\delta L^{a}}+\left(\frac{\delta \Sigma}{\delta \mathfrak{D}^{a}}+J^{a}\right) \frac{\delta \Sigma}{\delta T^{a}} \\
& -\frac{\delta \Sigma}{\delta \mathfrak{D}^{a}} X^{a \alpha}\left(\gamma_{\alpha \beta}\right) \epsilon^{\beta}+b^{a} \frac{\delta \Sigma}{\delta \bar{c}^{a}} \\
& +\left(\nabla \bar{c}^{a}\right) \frac{\delta \Sigma}{\delta b^{a}}+\left(\nabla K_{\mu}^{a}\right) \frac{\delta \Sigma}{\delta \Omega_{\mu}^{a}}+\left(\nabla Y^{a \alpha}\right) \frac{\delta \Sigma}{\delta X^{a \alpha}} \\
& \left.+\left(\nabla T^{a}\right) \frac{\delta \Sigma}{\delta J^{a}}+\left(\nabla L^{a}\right) \frac{\delta \Sigma}{\delta \Lambda^{a}}\right\} .
\end{aligned}
$$

From the Slavnov-Taylor identity (18), it follows that the so-called linearized operator $\mathcal{B}_{\Sigma}$ [3],

$$
\begin{aligned}
\mathcal{B}_{\Sigma}= & \int \mathrm{d}^{4} x\left\{\frac{\delta \Sigma}{\delta K_{\mu}^{a}} \frac{\delta}{\delta A_{\mu}^{a}}+\frac{\delta \Sigma}{\delta A_{\mu}^{a}} \frac{\delta}{\delta K_{\mu}^{a}}\right. \\
& +\Omega_{\mu}^{a} \frac{\delta}{\delta K_{\mu}^{a}}+\frac{\delta \Sigma}{\delta Y^{a \alpha}} \frac{\delta}{\delta \lambda^{a \alpha}} \\
& +\frac{\delta \Sigma}{\delta \lambda^{a \alpha}} \frac{\delta}{\delta Y^{a \alpha}}+X^{a \alpha} \frac{\delta}{\delta Y^{a \alpha}}+\frac{\delta}{\delta \lambda^{a \alpha}}\left(\gamma_{5}\right)_{\alpha \beta} \epsilon^{\beta} J^{a} \\
& +\frac{\delta \Sigma}{\delta L^{a}} \frac{\delta}{\delta c^{a}}+\frac{\delta \Sigma}{\delta c^{a}} \frac{\delta}{\delta L^{a}}
\end{aligned}
$$




$$
\begin{aligned}
& +\Lambda^{a} \frac{\delta}{\delta L^{a}}+\frac{\delta \Sigma}{\delta T^{a}} \frac{\delta}{\delta \mathfrak{D}^{a}}+\frac{\delta \Sigma}{\delta \mathfrak{D}^{a}} \frac{\delta}{\delta T^{a}} \\
& +J^{a} \frac{\delta}{\delta T^{a}}-X^{a \alpha}\left(\gamma_{\alpha \beta}\right) \epsilon^{\beta} \frac{\delta}{\delta \mathfrak{D}^{a}}+b^{a} \frac{\delta}{\delta \bar{c}^{a}} \\
& +\left(\nabla \bar{c}^{a}\right) \frac{\delta}{\delta b^{a}}+\left(\nabla K_{\mu}^{a}\right) \frac{\delta}{\delta \Omega_{\mu}^{a}}+\left(\nabla Y^{a \alpha}\right) \frac{\delta}{\delta X^{a \alpha}} \\
& \left.+\left(\nabla T^{a}\right) \frac{\delta}{\delta J^{a}}+\left(\nabla L^{a}\right) \frac{\delta}{\delta \Lambda^{a}}\right\},
\end{aligned}
$$

enjoys the following property:

$$
\mathcal{B}_{\Sigma} \mathcal{B}_{\Sigma}=\nabla
$$

so that $\mathcal{B}_{\Sigma}$ is nilpotent when acting on integrated functionals.

- The Landau gauge-fixing condition and the antighost equation [3]:

$$
\frac{\delta \Sigma}{\delta b^{a}}=\partial_{\mu} A_{\mu}^{a}, \quad \frac{\delta \Sigma}{\delta \bar{c}^{a}}+\partial_{\mu} \frac{\delta \Sigma}{\delta K_{\mu}^{a}}=0 .
$$

- The ghost Ward identity $[3,11]$ :

$$
G^{a}(\Sigma)=\Delta_{\text {class }}^{a}
$$

where

$$
G^{a}:=\int \mathrm{d}^{4} x\left[\frac{\delta}{\delta c^{a}}+\mathrm{gf}^{a b c} \bar{c}^{b} \frac{\delta}{\delta b^{c}}\right]
$$

and

$$
\begin{aligned}
\Delta_{\text {class }}^{a}= & \int \mathrm{d}^{4} x\left[\operatorname { g f } ^ { a b c } \left(K_{\mu}^{b} A_{\mu}^{c}-L^{b} c^{c}\right.\right. \\
& \left.\left.+T^{b} \mathfrak{D}^{a}-Y^{b \alpha} \lambda_{\alpha}^{c}\right)-\Lambda^{a}\right] .
\end{aligned}
$$

Notice that the breaking term $\Delta_{\text {class }}^{a}$ appearing on the righthand side of (22) is linear in the quantum fields. As such, $\Delta_{\text {class }}^{a}$ is a classical breaking, not affected by quantum corrections $[3,11]$.

- The equation of motion of the auxiliary field $\mathfrak{D}^{a}$ :

$$
\frac{\delta \Sigma}{\delta \mathfrak{D}^{a}}=\mathfrak{D}^{a}-J^{a}+\mathrm{gf}^{a b c} c^{b} T^{c}+Y^{a \alpha}\left(\gamma_{5}\right)_{\alpha \beta} \varepsilon^{\beta} .
$$

Again, being linear in the quantum fields, the right-hand side of (25) is a classical breaking.

- The linearly broken gluino Ward identity, namely:

$$
\left[\frac{\delta}{\delta T^{a}}+\left(\gamma_{5}\right)_{\alpha \beta} \varepsilon^{\beta} \frac{\delta}{\delta \lambda_{\alpha}^{a}}+\mathrm{gf}^{a b c}\left(c^{b} \frac{\delta}{\delta \mathfrak{D}^{c}}-T^{b} \frac{\delta}{\delta L^{c}}\right)\right] \Sigma=\tilde{\Delta}_{\text {class }}^{a}
$$

where $\tilde{\Delta}_{\text {class }}^{a}$ is a classical breaking

$$
\begin{aligned}
\tilde{\Delta}_{\text {class }}^{a}= & 3 \mathrm{gf}^{a b c} \bar{\epsilon}^{\alpha}\left(\gamma_{\mu}\right)_{\alpha \beta} \epsilon^{\beta} T^{b} A_{\mu}^{c}+\nabla T^{b}-\mathrm{gf}^{a b c} c^{b} J^{c} \\
& +\bar{\epsilon}^{\alpha}\left(\gamma_{\mu}\right)_{\alpha \eta}\left(\gamma_{5}\right)^{\eta \beta} \epsilon_{\beta}\left(\partial_{\mu} \bar{c}^{a}+K_{\mu}^{a}\right) .
\end{aligned}
$$

We notice, in particular, that the gluino Ward identity (26) follows by commuting the Slavnov-Taylor identity (18) with Eq. (25).

Before turning to the algebraic analysis of the most general invariant counterterm, let us spend a few words on the role of the auxiliary fields $\mathfrak{D}^{a}$, which we have introduced in the expression of the starting action $S_{\mathrm{SYM}}$, (4). As it is apparent from (4), the fields $\mathfrak{D}^{a}$ enter the action $S_{\mathrm{SYM}}$ only quadratically. As such, they do not play any role in the loop calculations. Though, they are needed in order to write down the Slavnov-Taylor identities (18), which are at the basis of the algebraic renormalization setup [3]. Here, we have two equivalent options. The first option is that of starting from the beginning by including the $\mathfrak{D}^{a}$ fields in the action, (4), as well as in the $Q$-transformations (6). In this case, the BRST operator $Q$ enjoys the important property

$Q^{2}=\nabla$,

which enables us to construct the Slavnov-Taylor identities in the way described before. The second option is that of not including the fields $\mathfrak{D}^{a}$ from the beginning; see, for instance, [6-8]. This means that the fields $\mathfrak{D}^{a}$ are absent in both the starting action and the $Q$-transformations. However, the BRST operator $Q$ does not display now the property (28). Instead, one has

$Q^{2}=\nabla+$ eqs. of motion.

In this case, in order to establish the Slavnov-Taylor identities, an additional care has to be taken. The presence of terms proportional to the equations of motion in (29) requires the introduction of terms which are quadratic in the BRST sources [6-8]. These terms are precisely of the same kind of $\mathfrak{D}^{a} \mathfrak{D}^{a}$. At the end of this second procedure, one is able to write down Slavnov-Taylor identities which are exactly of the same type of (18) [6-8], so that both options give the same results for the characterization of the invariant counterterm.

\subsection{Algebraic characterization of the invariant counterterm and renormalizability of the $\mathcal{N}=1$ Super-Yang-Mills}

In order to determine the most general invariant counterterm which can be freely added to each order, we follow the algebraic renormalization framework [3] and perturb the complete action $\Sigma$ by adding an integrated local polynomial in the fields and sources with dimension four and vanishing ghost number, $\Sigma_{\text {count }}$, and we require that the perturbed action, $\left(\Sigma+\omega \Sigma_{\text {count }}\right)$, where $\omega$ is an infinitesimal expansion 
parameter, obeys the same Ward identities as fulfilled by $\Sigma$ to the first order in the parameter $\omega$, namely

$$
\begin{aligned}
& \mathcal{S}\left(\Sigma+\omega \Sigma_{\text {count }}\right)=0+O\left(\omega^{2}\right), \\
& \frac{\delta\left(\Sigma+\omega \Sigma_{\text {count }}\right)}{\delta b^{a}}=\partial_{\mu} A_{\mu}^{a}+O\left(\omega^{2}\right), \\
& \left(\frac{\delta}{\delta \bar{c}^{a}}+\partial_{\mu} \frac{\delta}{\delta K_{\mu}^{a}}\right)\left(\Sigma+\omega \Sigma_{\text {count }}\right)=0+O\left(\omega^{2}\right), \\
& G^{a}\left(\Sigma+\omega \Sigma_{\text {count }}\right)=\Delta_{\text {class }}^{a}+O\left(\omega^{2}\right), \\
& \frac{\delta\left(\Sigma+\omega \Sigma_{\text {count }}\right)}{\delta \mathfrak{D}^{a}}=\mathfrak{D}^{a}-J^{a}+\mathrm{gf}^{a b c} c^{b} T^{c} \\
& \quad+Y^{a \alpha}\left(\gamma_{5}\right)_{\alpha \beta} \varepsilon^{\beta}+O\left(\omega^{2}\right), \\
& {\left[\frac{\delta}{\delta T^{a}}+\left(\gamma_{5}\right)_{\alpha \beta} \varepsilon^{\beta} \frac{\delta}{\delta \lambda_{\alpha}^{a}}+\mathrm{gf}^{a b c}\left(c^{b} \frac{\delta}{\delta \mathfrak{D}^{c}}-T^{b} \frac{\delta}{\delta L^{c}}\right)\right]} \\
& \quad \times\left(\Sigma+\omega \Sigma_{\text {count }}\right)=\tilde{\Delta}_{\text {class }}^{a}+O\left(\omega^{2}\right) .
\end{aligned}
$$

To the first order in the expansion parameter $\omega,(30),(31)$, (32), (33), and (34) give rise to the following constraints:

$\mathcal{B}_{\Sigma}\left(\Sigma_{\text {count }}\right)=0$

$\frac{\delta}{\delta b^{a}} \Sigma_{\text {count }}=0, \quad\left(\frac{\delta}{\delta \bar{c}^{a}}+\partial_{\mu} \frac{\delta}{\delta K_{\mu}^{a}}\right) \Sigma_{\text {count }}=0$,

$G^{a} \Sigma_{\text {count }}=0$,

$\frac{\delta}{\delta \mathfrak{D}^{a}} \Sigma_{\text {count }}=0$

and

$\left[\frac{\delta}{\delta T^{a}}+\left(\gamma_{5}\right)_{\alpha \beta} \varepsilon^{\beta} \frac{\delta}{\delta \lambda_{\alpha}^{a}}+\mathrm{gf}^{a b c}\left(c^{b} \frac{\delta}{\delta \mathfrak{D}^{c}}-T^{b} \frac{\delta}{\delta L^{c}}\right)\right]$

$$
\times \Sigma_{\text {count }}=0 \text {, }
$$

where $\mathcal{B}_{\Sigma}$ stands for the linearized operator of (19). The first condition, (35), tells us that $\Sigma_{\text {count }}$ belongs to the cohomology of the operator $\mathcal{B}_{\Sigma}$ in the space of the local integrated polynomials in the fields and external sources of dimension bounded by four. From the general results on the cohomology of Yang-Mills theories, see [3] and references therein, it follows that $\Sigma_{\text {count }}$ can be parametrized as follows:

$\Sigma_{\text {count }}=a_{0} S_{\mathrm{SYM}}+\mathcal{B}_{\Sigma} \Delta^{(-1)}$.

where $a_{0}$ is a free coefficient and $\Delta^{(-1)}$ stands for the most general integrated local polynomial in the fields and sources, with ghost number -1 and dimension 3 .
From Table 1, the most general expression for $\Delta^{(-1)}$ can be written as

$$
\begin{aligned}
\Delta^{(-1)}= & \int \mathrm{d}^{4} x\left\{a_{1} \partial_{\mu} \bar{c}^{a} A_{\mu}^{a}+a_{2} K_{\mu}^{a} A_{\mu}^{a}+a_{3} T^{a} \partial_{\mu} A_{\mu}^{a}\right. \\
& +a_{4} b^{a} \bar{c}^{a}+a_{5} b^{a} T^{a}+a_{6} \mathfrak{D}^{a} \bar{c}^{a} \\
& +a_{7} J^{a} T^{a}+a_{8} \lambda^{a \alpha} Y_{a \alpha}+a_{9} Y^{a \alpha}\left(\gamma_{5}\right)_{\alpha \beta} \epsilon^{\beta} T^{a} \\
& +a_{10} \mathrm{gf}^{a b c} \bar{c}^{a} \bar{c}^{b} c^{c}+a_{11} J^{a} \bar{c}^{a} \\
& +a_{12} \bar{c}^{a} \epsilon^{\alpha}\left(\gamma_{5}\right)_{\alpha \beta} Y^{a \beta}+a_{13} \mathrm{gf}^{a b c} T^{a} T^{b} c^{c} \\
& \left.+a_{14} \mathfrak{D}^{a} T^{a}+a_{15} \mathrm{gf}^{a b c} c^{a} \bar{c}^{b} T^{c}+a_{16} c^{a} L^{a}\right\}
\end{aligned}
$$

with $a_{i}(i=1$ to 16$)$ being arbitrary coefficients. It is worth to point out that, according to Table 1 , the ultraviolet dimension of both ghost and antighost fields, $(c, \bar{c})$, has been chosen to be equal to 1 . This feature turns out to be very helpful, as enables us to assign positive ultraviolet dimension $1 / 2$ to the supersymmetric parameter $\epsilon$, a property which greatly simplifies the analysis of the invariant counterterm $\Sigma_{\text {count }}$.

From (36), (37), (38), and (39), it follows that

$$
\begin{aligned}
& a_{1}=a_{2}, \quad a_{14}=-\frac{a_{0}}{2}, \quad a_{9}=\left(\frac{a_{0}}{2}-a_{8}\right) \quad \text { and } \\
& a_{3}=a_{4}=a_{5}=a_{6}=a_{7}=a_{10}=a_{11}=a_{12}=0, \\
& a_{13}=a_{15}=a_{16}=a_{17}=a_{18}=a_{19}=0,
\end{aligned}
$$

leading to

$$
\begin{aligned}
\Delta^{(-1)}= & \int \mathrm{d}^{4} x\left\{a_{1}\left(\partial_{\mu} \bar{c}^{a}+K_{\mu}^{a}\right) A_{\mu}^{a}+a_{8} Y^{a \alpha} \lambda_{\alpha}^{a}\right. \\
& \left.+\left(\frac{a_{0}}{2}-a_{8}\right) Y^{a \alpha}\left(\gamma_{5}\right)_{\alpha \beta} \epsilon^{\beta} T^{a}-\frac{a_{0}}{2} \mathfrak{D}^{a} T^{a}\right\} .
\end{aligned}
$$

Therefore, for the exact part of (40), i.e. $\mathcal{B}_{\Sigma} \Delta^{(-1)}$, we get

$$
\begin{aligned}
\mathcal{B}_{\Sigma} \Delta^{(-1)}= & \int \mathrm{d}^{4} x\left\{a_{1}\left(\frac{\delta \Sigma}{\delta A_{\mu}^{a}}+\Omega_{\mu}^{a}+\partial_{\mu} b^{a}\right) A_{\mu}^{a}\right. \\
& +a_{8}\left(\frac{\delta \Sigma}{\delta \lambda_{\alpha}^{a}}+X^{a \alpha}\right)\left(\lambda_{\alpha}^{a}-\left(\gamma_{5}\right)_{\alpha \beta} \epsilon_{\beta} T^{a}\right) \\
& +\frac{a_{0}}{2}\left(\frac{\delta \Sigma}{\delta \lambda_{\alpha}^{a}}+X^{a \alpha}\right)\left(\gamma_{5}\right)_{\alpha \beta} \epsilon^{\beta} T^{a} \\
& -\frac{a_{0}}{2}\left(\frac{\delta \Sigma}{\delta \mathfrak{D}^{a}}+J^{a}\right)\left(\mathfrak{D}^{a}-Y^{a \alpha}\left(\gamma_{5}\right)_{\alpha \beta} \epsilon^{\beta}\right) \\
& -a_{8}\left(\frac{\delta \Sigma}{\delta \mathfrak{D}^{a}}+J^{a}\right) Y^{a \alpha}\left(\gamma_{5}\right)_{\alpha \beta} \epsilon^{\beta} \\
& -a_{1} \bar{c}^{a} \frac{\delta \Sigma}{\delta \bar{c}^{a}}-a_{1} K_{\mu}^{a} \frac{\delta \Sigma}{\delta K_{\mu}^{a}}+a_{8} Y^{a \alpha} \frac{\delta \Sigma}{\delta Y^{a \alpha}} \\
& \left.+\frac{a_{0}}{2} T^{a} \frac{\delta \Sigma}{\delta T^{a}}\right\},
\end{aligned}
$$


Table 1 Quantum numbers of all fields and sources. "A" stands for anticommuting, while "C" stands for commuting

\begin{tabular}{lllllllllllllllll}
\hline & $A$ & $\lambda$ & $\mathfrak{D}$ & $c$ & $\bar{c}$ & $b$ & $K$ & $\Omega$ & $\Lambda$ & $T$ & $J$ & $L$ & $Y$ & $X$ & $\epsilon$ & $\bar{\epsilon}$ \\
\hline Dim & 1 & $\frac{3}{2}$ & 2 & 1 & 1 & 2 & 2 & 3 & 3 & 1 & 2 & 2 & $\frac{3}{2}$ & $\frac{5}{2}$ & $\frac{1}{2}$ & $\frac{1}{2}$ \\
Ghost\# & 0 & 0 & 0 & 1 & -1 & 0 & -1 & 0 & -1 & -1 & 0 & -2 & -1 & 0 & 1 & 1 \\
Nature & $\mathrm{C}$ & $\mathrm{A}$ & $\mathrm{C}$ & $\mathrm{A}$ & $\mathrm{A}$ & $\mathrm{C}$ & $\mathrm{A}$ & $\mathrm{C}$ & $\mathrm{A}$ & $\mathrm{A}$ & $\mathrm{C}$ & $\mathrm{C}$ & $\mathrm{C}$ & $\mathrm{A}$ & $\mathrm{C}$ & $\mathrm{C}$ \\
\hline
\end{tabular}

yielding the final form of the most general invariant counterterm

$$
\begin{aligned}
\Sigma_{\mathrm{count}}= & \int \mathrm{d}^{4} x\left\{\frac{a_{0}}{4} F_{\mu \nu}^{a} F_{\mu \nu}^{a}+a_{1} \frac{\delta \Sigma_{\mathrm{SYM}}}{\delta A_{\mu}^{a}} A_{\mu}^{a}\right. \\
& +\frac{\left(a_{0}-2 a_{8}\right)}{2} \bar{\lambda}^{a \alpha}\left(\gamma_{\mu}\right)_{\alpha \beta} D_{\mu}^{a b} \lambda^{b \beta} \\
& +a_{1}\left(\partial_{\mu} \bar{c}^{a}+K_{\mu}^{a}\right) \partial_{\mu} c^{a}+\left(a_{1}+a_{8}\right) \\
& \times \bar{\epsilon}^{\alpha}\left(\gamma_{\mu}\right)_{\alpha \beta} \lambda^{a \beta}\left(\partial_{\mu} \bar{c}^{a}+K_{\mu}^{a}\right) \\
& +\left(a_{0}-2 a_{8}\right) \bar{\epsilon}^{\alpha}\left(\gamma_{\mu}\right)_{\alpha \beta}\left(\gamma_{5}\right)^{\beta \eta} T^{a} D_{\mu}^{a b} \lambda_{\eta}^{b} \\
& -a_{1} \mathrm{gf}{ }^{a b c} T^{a} \bar{\epsilon}^{\alpha}\left(\gamma_{\mu}\right)_{\alpha \beta}\left(\gamma_{5}\right)^{\beta \eta} \lambda_{\eta}^{b} A_{\mu}^{c} \\
& -a_{1} \bar{\epsilon}^{\alpha}\left(\gamma_{\mu}\right)_{\alpha \beta} \epsilon^{\beta} A_{\mu}^{a} L^{a} \\
& +\left(a_{8}-\frac{a_{0}}{2}\right) \bar{\epsilon}^{\alpha}\left(\gamma_{\mu}\right)_{\alpha \beta} \epsilon^{\beta} T^{a} D_{\mu}^{a b} T^{b} \\
& +\left(\frac{a_{0}}{2}-a_{8}\right)\left(Y^{a \alpha}\left(\gamma_{5}\right)_{\alpha \beta} \epsilon^{\beta}\right)^{2} \\
& -\frac{1}{2}\left(a_{1}+a_{8}\right) Y^{a \alpha}\left(\sigma_{\mu \nu}\right)_{\alpha \beta} \epsilon^{\beta}\left(\partial_{\mu} A_{\nu}^{a}-\partial_{\nu} A_{\mu}^{a}\right) \\
& \left.-\left(a_{1}+\frac{a_{8}}{2}\right) \mathrm{gf}^{a b c} Y^{a \alpha}\left(\sigma_{\mu \nu}\right)_{\alpha \beta} \epsilon^{\beta} A_{\mu}^{b} A_{\nu}^{c}\right\} .
\end{aligned}
$$

One sees that $\Sigma_{\text {count }}$ contains three arbitrary coefficients, $a_{0}, a_{1}$, and $a_{8}$, which will identify the renormalization factors of all fields, sources, and coupling constant. To complete the analysis of the algebraic renormalization of the model, we need to show that the counterterm $\Sigma_{\text {count }}$ can be reabsorbed into the starting action $\Sigma$ through a redefinition of the fields and parameters $\{\phi\}, \phi=(A, \lambda, b, c, \bar{c}, \mathfrak{D}, \epsilon)$, of the sources $\{S\}, S=(K, \Omega, \Lambda, T, J, L, Y, X)$, and coupling constant $g$, namely

$\Sigma(\phi, S, g)+\omega \Sigma_{\text {count }}(\phi, S, g)=\Sigma\left(\phi_{0}, S_{0}, g_{0}\right)+O\left(\omega^{2}\right)$,

where $\left(\phi_{0}, S_{0}, g_{0}\right)$ stand for the so-called bare fields, sources, and coupling constant:

$\phi_{0}=Z_{\phi}^{1 / 2} \phi, \quad S_{0}=Z_{S} S, \quad g_{0}=Z_{g} g$,

and the renormalization factors $Z$ can be written as

$Z_{\phi}^{1 / 2}=\left(1+\omega z_{\phi}\right)^{1 / 2}=1+\omega \frac{z_{\phi}}{2}+O\left(\omega^{2}\right)$,

$Z_{S}=1+\omega z_{S}, \quad Z_{g}=1+\omega z_{g}$.

Moreover, in the present case, a little care has to be taken with the potential mixing of quantities which have the same quantum numbers. In fact, from equation (45) one can easily notice that the field $\lambda^{a \alpha}$ and the combination $\gamma_{5} \epsilon T^{a}$ have the same dimension and quantum numbers as well as the field $\mathfrak{D}^{a}$ and the combination $Y^{a} \gamma_{5} \epsilon$, as can be checked from Table 1. As a consequence, these quantities can mix at the quantum level, a well-known property of renormalization theory. This feature can be properly taken into account by writing the renormalization of the fields $\lambda$ and $\mathfrak{D}$ in matrix form, i.e.

$\lambda_{0}^{a \alpha}=Z_{\lambda}^{1 / 2} \lambda^{a \alpha}+\omega z_{1} T^{a}\left(\gamma_{5}\right)^{\alpha \beta} \varepsilon_{\beta}$

and

$\mathfrak{D}_{0}^{a}=Z_{\mathfrak{D}}^{1 / 2} \mathfrak{D}^{a}+\omega z_{2} Y^{a \alpha}\left(\gamma_{5}\right)_{\alpha \beta} \varepsilon^{\beta}$,

while the remaining fields, sources, and parameters still obey (3.2).

From direct inspection of equation (47), the renormalization factors of all fields, sources, and parameters are given by

$$
\begin{aligned}
Z_{A}^{1 / 2} & =1+\omega\left(\frac{a_{0}}{2}+a_{1}\right), \\
Z_{g} & =1-\omega \frac{a_{0}}{2}, \\
Z_{\lambda}^{1 / 2} & =1+\omega\left(\frac{a_{0}}{2}-a_{8}\right),
\end{aligned}
$$

while the remaining factors are

$$
\begin{aligned}
Z_{T} & =Z_{g}^{-1 / 2} Z_{A}^{1 / 4}, \\
Z_{\varepsilon} & =Z_{g}^{1 / 2} Z_{A}^{-1 / 4}, \\
Z_{Y} & =Z_{g}^{-1 / 2} Z_{A}^{1 / 4} Z_{\lambda}^{-1 / 2}, \\
Z_{b}^{1 / 2} & =Z_{A}^{-1 / 2}, \\
Z_{L} & =Z_{A}^{1 / 2}, \\
Z_{c}^{1 / 2} & =Z_{\bar{c}}^{1 / 2}=Z_{K}=Z_{g}^{-1 / 2} Z_{A}^{-1 / 4}, \\
Z_{\Lambda} & =Z_{g}^{1 / 2} Z_{A}^{1 / 4}, \\
Z_{J} & =1 \\
Z_{\mathfrak{D}} & =1 \\
Z_{X} & =Z_{\lambda}^{-1 / 2}, \\
Z_{\Omega} & =Z_{A}^{-1 / 2}
\end{aligned}
$$

and

$z_{1}=-z_{2}=a_{8}-\frac{a_{0}}{2}$ 
We have thus completed the all-order proof of the algebraic renormalization of $\mathcal{N}=1$ supersymmetric Yang-Mills theories. A few remarks are in order. Three independent parameters, $a_{0}, a_{1}, a_{8}$, are needed to renormalize the theory. According to (52), these parameters correspond to the renormalization of the gauge coupling constant $g$, of the gauge field $A_{\mu}^{a}$ and of the gluino $\lambda^{a \alpha}$. The renormalization constants of all other fields, sources, and parameters can be written down as suitable combinations of $Z_{g}, Z_{A}, Z_{\lambda}$, as expressed by (53), (54). We remark that the celebrated non-renormalization theorem of the gluon-ghost-antighost vertex of the Landau gauge [3,11], i.e. $Z_{c}^{1 / 2} Z_{\bar{c}}^{1 / 2} Z_{g} Z_{A}^{1 / 2}=1$, remains valid in the supersymmetric version of the theory. Moreover, although belonging to the same multiplet, (52) suggest that the renormalization constant of the gauge field, $Z_{A}$, turns out to be different from that of the gluino, $Z_{\lambda}$. That this will be in fact the case will be shown in the next section, where the explicit three-loop expression of $Z_{A}, Z_{\lambda}$ will be reported.

\section{Three-loop calculation of the renormalization factors $Z_{A}$ and $Z_{\lambda}$ and check of the non-renormalization theorem of the gluon-ghost-antighost vertex}

We explicitly computed the wave-function renormalization constants for the bosonic and fermionic degrees of freedom $Z_{A}, Z_{\lambda}$, and $Z_{c}$ and the gauge coupling renormalization constant $Z_{g}$ up to three loops in perturbation theory. As renormalization scheme we used the minimal subtraction scheme with dimensional reduction [15] (DRED) as regulator. Such renormalization scheme is commonly denoted $\overline{\mathrm{DR}}$. Let us mention that we applied DRED in the component field formalism and implemented its mathematical consistent formulation $[16,17]$. It is well known that DRED in this formulation breaks supersymmetry in higher orders of perturbation theory [18]. Nevertheless, for a supersymmetric Yang-Mills theory it has been proven explicitly that DRED preserves supersymmetry up to three loops $[19,20]$.

The advantage of this scheme is that all ultraviolet (UV) counterterms are polynomial both in external momenta and masses $[21,22]$. The most effective approach is its use in combination with multiplicative renormalization. This amounts in general to solving recursively the equation

$Z_{a}=1-K_{\varepsilon}\left[\Gamma_{a}\left(p^{2}\right) Z_{a}\right]$,

where $K_{\varepsilon}[f(\varepsilon)]$ stands for the singular part of the Laurent expansion of $f(\varepsilon)$ in $\varepsilon$ around $\varepsilon=0$. $\Gamma_{a}\left(p^{2}\right)$ denotes the renormalized Green function with only one external momentum $p^{2}$ kept non-zero. $Z_{a}$ denotes the renormalization constant associated with the Green function $\Gamma_{a}$. In this case, the renormalization of $\Gamma_{a}$ through $(l+1)$-loop order requires the renormalization of the Lagrangian parameters like couplings, masses, gauge parameters, etc. up to $l$-loop order. For the present calculation we considered the renormalization of the Green functions corresponding to the gauge boson propagator, its ghost and its Majorana superpartner propagators and the vertices containing ghost-gauge boson and Majorana fermion-gauge boson interactions.

For the explicit calculation of Feynman diagrams up to three-loop order, we used a well-tested chain of programs: QGRAF [23] generates all contributing Feynman diagrams. The output is passed via $q 2 e[24,25]$, which transforms Feynman diagrams into Feynman amplitudes, to exp [24,25] that generates FORM [26] code. The latter is processed by MINCER [27] which computes analytically massless propagator diagrams up to three loops and outputs the $\epsilon$ expansion of the result. Here, $\epsilon=(4-d) / 2$ is the regulator of Dimensional Regularization with $d$ being the space-time dimension used for the evaluation of the momentum integrals.

We performed all the calculations in a linear gauge and only in the last step specified the results to the Landau gauge. This procedure allows us to check explicitly the gauge independence of the gauge coupling renormalization constant. In our setup, the gauge parameter $\xi$ is defined through the gauge boson propagator

$$
D_{\mu \nu}^{A}=-i \frac{g_{\mu \nu}-(1-\xi) \frac{q_{\mu} q_{v}}{q^{2}}}{q^{2}+i \varepsilon}
$$

The three-loop expression for the wave-function renormalization constant of the Majorana field reads

$$
\begin{aligned}
Z_{\lambda}= & 1-\frac{1}{\epsilon}\left(\frac{\alpha}{4 \pi}\right) C_{A} \xi \\
& +\left(\frac{\alpha}{4 \pi}\right)^{2} C_{A}^{2}\left[\frac{1}{4 \epsilon^{2}} 3 \xi(1+\xi)-\frac{1}{8 \epsilon}\left(3+8 \xi+\xi^{2}\right)\right] \\
& +\left(\frac{\alpha}{4 \pi}\right)^{3} C_{A}^{3}\left[-\frac{1}{8 \epsilon^{3}} \xi\left(9+9 \xi+4 \xi^{2}\right)\right. \\
& +\frac{1}{4 \epsilon^{2}}\left(3+11 \xi+7 \xi^{2}+\xi^{3}\right) \\
& +\frac{1}{96 \epsilon}\left(66-108 Z_{3}-3 \xi\left(53+8 Z_{3}\right)\right. \\
& \left.\left.-3 \xi^{2}\left(13+4 Z_{3}\right)-10 \xi^{3}\right)\right]
\end{aligned}
$$

Here $Z_{3}=\zeta_{3}$ is the Riemann $\zeta$-function, $\alpha=\frac{g}{4 \pi}$ and $C_{A}$ is the quadratic Casimir invariant in the adjoint representation. In the special case of the Landau gauge, for which $\xi=0$, it reduces to

$$
\begin{aligned}
Z_{\lambda}= & 1-\left(\frac{\alpha}{4 \pi}\right)^{2} \frac{3}{8 \epsilon} C_{A}^{2} \\
& +\left(\frac{\alpha}{4 \pi}\right)^{3} C_{A}^{3}\left[+\frac{3}{4 \epsilon^{2}}+\frac{1}{48 \epsilon}\left(33-54 Z_{3}\right)\right] .
\end{aligned}
$$

For the three-loop expression of the wave-function renormalization constant of the gauge boson, we obtained

$$
Z_{A}=1+\left(\frac{\alpha}{4 \pi}\right) \frac{1}{2 \epsilon} C_{A}(3-\xi)
$$




$$
\begin{aligned}
& +\left(\frac{\alpha}{4 \pi}\right)^{2} C_{A}^{2}\left[\frac{1}{8 \epsilon^{2}}\left(-9-3 \xi+2 \xi^{2}\right) \frac{1}{16 \epsilon}\left(27-11 \xi-2 \xi^{2}\right)\right] \\
& +\left(\frac{\alpha}{4 \pi}\right)^{3} C_{A}^{3}\left[\frac{1}{16 \epsilon^{3}}\left(27+9 \xi-2 \xi^{3}\right)\right. \\
& +\frac{1}{96 \epsilon^{2}}\left(-369-39 \xi+60 \xi^{2}+14 \xi^{3}\right) \\
& +\frac{1}{96 \epsilon}\left(533-7 \xi^{3}-114 Z_{3}-3 \xi^{2}\left(11+2 Z_{3}\right)\right. \\
& \left.\left.-\xi\left(113+24 Z_{3}\right)\right)\right] .
\end{aligned}
$$

It is an easy exercise to obtain its expression for the Landau gauge,

$$
\begin{aligned}
Z_{A}= & +\left(\frac{\alpha}{4 \pi}\right) \frac{3 C_{A}}{2 \epsilon}+\left(\frac{\alpha}{4 \pi}\right)^{2} C_{A}^{2}\left[-\frac{9}{8 \epsilon^{2}}+\frac{27}{16 \epsilon}\right] \\
& +\left(\frac{\alpha}{4 \pi}\right)^{3} C_{A}^{3}\left[\frac{27}{16 \epsilon^{3}}-\frac{123}{32 \epsilon^{2}}+\frac{1}{96 \epsilon}\left(533-114 Z_{3}\right)\right] .
\end{aligned}
$$

The expression for the three-loop wave-function renormalization constant of the ghost is given by

$$
\begin{aligned}
Z_{c}= & 1+\left(\frac{\alpha}{4 \pi}\right) \frac{1}{4 \epsilon} C_{A}(3-\xi) \\
& +\left(\frac{\alpha}{4 \pi}\right)^{2} C_{A}^{2}\left[\frac{3}{32 \epsilon^{2}}\left(-9+\xi^{2}\right)+\frac{1}{32 \epsilon}(21+\xi)\right] \\
& +\left(\frac{\alpha}{4 \pi}\right)^{3} C_{A}^{3}\left[\frac{1}{128 \epsilon^{3}}\left(189+9 \xi-9 \xi^{2}-5 \xi^{3}\right)\right. \\
& +\frac{1}{384 \epsilon^{2}}\left(-891+12 \xi+39 \xi^{2}+8 \xi^{3}\right) \\
& \left.+\frac{1}{192 \epsilon}\left(139-3 \xi^{3}+114 Z_{3}+6 \xi^{2}\left(-1+Z_{3}\right)+24 \xi Z_{3}\right)\right] .
\end{aligned}
$$

The simplified formula for the case of the Landau gauge reads

$$
\begin{aligned}
Z_{c}= & 1+\left(\frac{\alpha}{4 \pi}\right) \frac{3}{4 \epsilon} C_{A}+\left(\frac{\alpha}{4 \pi}\right)^{2} C_{A}^{2}\left[-\frac{27}{32 \epsilon^{2}}+\frac{21}{32 \epsilon}\right] \\
& +\left(\frac{\alpha}{4 \pi}\right)^{3} C_{A}^{3}\left[\frac{189}{128 \epsilon^{3}}-\frac{297}{128 \epsilon^{2}}+\frac{1}{192 \epsilon}\left(139+114 Z_{3}\right)\right] .
\end{aligned}
$$

Our results for the three-loop renormalization constant of the gauge coupling completely agree with the previous calculations of Refs. [20,28,29]. For convenience of the reader we quote them below

$$
\begin{aligned}
Z_{g}= & 1-\left(\frac{\alpha}{4 \pi}\right) \frac{3}{2 \epsilon} C_{A}+\left(\frac{\alpha}{4 \pi}\right)^{2} C_{A}^{2}\left[\frac{27}{8 \epsilon^{2}}-\frac{3}{2 \epsilon}\right] \\
& +\left(\frac{\alpha}{4 \pi}\right)^{3} C_{A}^{3}\left[-\frac{135}{16 \epsilon^{3}}+\frac{33}{4 \epsilon^{2}}-\frac{7}{2 \epsilon}\right] .
\end{aligned}
$$

Using (60), (62), and (63) one can immediately test the nonrenormalization of the gluon-ghost-antighost vertex, given in (53), i.e. $Z_{g} Z_{A}^{1 / 2} Z_{c}=1$.

\section{Conclusion}

In this work the issue of the renormalization of $\mathcal{N}=1$ Super Yang-Mills theory has been addressed in the Wess-Zumino gauge, by employing the Landau condition. Following the setup already outlined by the authors [5-10], the renormalization of the theory has been investigated within the Algebraic Renormalization framework [3], through BRST cohomology tools.

Our main result is summarized by (52), (53). In the Landau gauge, only three renormalization factors, $Z_{g}, Z_{A}, Z_{\lambda}$, are needed in order to renormalize the theory. The renormalization constants of all other fields can be expressed as suitable combinations of $Z_{g}, Z_{A}, Z_{\lambda}$, as displayed by (53). Moreover, although belonging to the same multiplet, the renormalization constant of the gauge field, $Z_{A}$, turns out to be different from that of the gluino, $Z_{\lambda}$, as explicitly checked through the three-loop computations; see (58) and (60). As already mentioned, this feature is due to the use of the Wess-Zumino gauge, in which the supersymmetry is realized in a non-linear way. Further, the non-renormalization theorem of the gluonghost-antighost vertex has been shown to remain valid in $\mathcal{N}=1$ Super Yang-Mills.

Finally, although we have limited ourselves to consider only the case of pure $\mathcal{N}=1$ Super Yang-Mills theory, the inclusion of matter fields can be done straightforwardly. Let us also point out that the non-renormalization of the gluonghost-antighost vertex remains valid in presence of matter fields, as a consequence of the ghost Ward identity, (22), which still holds in presence of matter [3,6-8].

Acknowledgments The Conselho Nacional de Desenvolvimento Científico e Tecnológico (CNPq-Brazil), the Faperj, Fundação de Amparo à Pesquisa do Estado do Rio de Janeiro, the Latin American Center for Physics (CLAF), the SR2-UERJ, the Coordenação de Aperfeiçoamento de Pessoal de Nível Superior (CAPES) are gratefully acknowledged. LM is supported by the DFG through SFB/TR 9.

Open Access This article is distributed under the terms of the Creative Commons Attribution License which permits any use, distribution, and reproduction in any medium, provided the original author(s) and the source are credited.

Funded by $\mathrm{SCOAP}^{3}$ / License Version CC BY 4.0.

\section{Appendix A: Notations and conventions in Euclidean space-time}

Units: $\hbar=c=1$.

Euclidean metric: $\delta_{\mu \nu}=\operatorname{diag}(+,+,+,+)$.

Wick rotations: $X_{0} \rightarrow-i X_{4} \Rightarrow \partial_{0} \rightarrow+i \partial_{4}, A_{0} \rightarrow+i A_{4}$ Gamma matrices:

$$
\gamma_{4}=\left(\begin{array}{ll}
0 & \mathbb{1} \\
\mathbb{1} & 0
\end{array}\right), \quad \gamma_{k}=-i\left(\begin{array}{cc}
0 & \sigma_{k} \\
-\sigma_{k} & 0
\end{array}\right) \text {. }
$$




\section{Pauli matrices:}

$$
\begin{aligned}
\sigma_{4} & =\left(\begin{array}{ll}
1 & 0 \\
0 & 1
\end{array}\right), \quad \sigma_{1}=\left(\begin{array}{ll}
0 & 1 \\
1 & 0
\end{array}\right), \quad \sigma_{2}=\left(\begin{array}{cc}
0 & -i \\
i & 0
\end{array}\right), \\
\sigma_{3} & =\left(\begin{array}{cc}
1 & 0 \\
0 & -1
\end{array}\right) .
\end{aligned}
$$

The Gamma matrices obey the following properties:

$$
\begin{aligned}
& \gamma_{\mu}=\gamma_{\mu}^{\dagger} \\
& \left\{\gamma_{\mu}, \gamma_{\nu}\right\}=2 \delta_{\mu \nu}
\end{aligned}
$$

We also define the $\gamma_{5}$ matrix as

$$
\gamma_{5}=\gamma_{4} \gamma_{1} \gamma_{2} \gamma_{3}=\left(\begin{array}{cc}
\mathbb{1} & 0 \\
0 & -\mathbb{1}
\end{array}\right)
$$

with the following properties:

$\left\{\gamma_{5}, \gamma_{\mu}\right\}=0, \quad\left(\gamma_{5}\right)^{2}=\mathbb{1}, \quad \gamma_{5}^{\dagger}=\gamma_{5}$

The charge conjugation matrix is

$\mathcal{C}=\gamma_{4} \gamma_{2}=i\left(\begin{array}{cc}\sigma_{2} & 0 \\ 0 & -\sigma_{2}\end{array}\right)$

with the following properties:

$$
\mathcal{C}^{-1}=-\mathcal{C}=\mathcal{C}^{T}, \quad \mathcal{C}^{-1} \gamma_{\mu} \mathcal{C}=-\gamma_{\mu}^{T}
$$

The $\sigma^{\mu \nu}$ tensor is defined as

$$
\left(\sigma_{\mu \nu}\right)_{\alpha}^{\beta} \equiv \frac{1}{2}\left[\gamma_{\mu}, \gamma_{\nu}\right]_{\alpha}^{\beta}
$$

and has the property $\sigma_{\mu \nu}^{\dagger}=-\sigma_{\mu \nu}$.

\section{Majorana fermions:}

The Majorana condition reads

$$
\lambda^{\mathcal{C}}=\lambda=\mathcal{C} \bar{\lambda}^{T} \Longleftrightarrow \bar{\lambda}=\lambda^{T} \mathcal{C},
$$

leading to the following relations:

$\bar{\lambda} \gamma_{\mu} \epsilon=\bar{\epsilon} \gamma_{\mu} \lambda$ and $\bar{\lambda} \gamma_{\mu} \gamma_{5} \epsilon=-\bar{\epsilon} \gamma_{\mu} \gamma_{5} \lambda$.

\section{Fierz identity (in Euclidean space-time):}

$$
\begin{aligned}
\epsilon_{1} \bar{\epsilon}_{2}= & \frac{1}{4}\left(\bar{\epsilon}_{2} \epsilon_{1}\right) \mathbb{1}+\frac{1}{4}\left(\bar{\epsilon}_{2} \gamma_{5} \epsilon_{1}\right) \gamma_{5}+\frac{1}{4}\left(\bar{\epsilon}_{2} \gamma_{\mu} \epsilon_{1}\right) \gamma_{\mu} \\
& -\frac{1}{4}\left(\bar{\epsilon}_{2} \gamma_{\mu} \gamma_{5} \epsilon_{1}\right) \gamma_{\mu} \gamma_{5}-\frac{1}{8}\left(\bar{\epsilon}_{2} \sigma_{\mu \nu} \epsilon_{1}\right) \sigma_{\mu \nu} .
\end{aligned}
$$

\section{Indices notations:}

- The Lorentz indices: $\mu, v, \rho, \sigma, \lambda \in\{1,2,3,4\}$;

- The spinor indices: $\alpha, \beta, \gamma, \delta, \eta \in\{1,2,3,4\}$;

- The $S U(N)$ group indices: $a, b, c, d, e \in\left\{1, \ldots, N^{2}-\right.$ $1\}$.

\section{References}

1. D. Amati, K. Konishi, Y. Meurice, G.C. Rossi, G. Veneziano, Phys. Rep. 162, 169 (1988)

2. S.J. Gates, M.T. Grisaru, M. Rocek, W. Siegel, Front. Phys. 58, 1 (1983). [hep-th/0108200]

3. O. Piguet, S.P. Sorella, Lect. Notes Phys. M 28, 1 (1995)

4. W. Hollik, D. Stockinger, Eur. Phys. J. C 20, 105 (2001). [hep-ph/ 0103009]

5. P.L. White, Class. Quantum Gravity 9, 1663 (1992)

6. N. Maggiore, Int. J. Mod. Phys. A 10, 3781 (1995). [hep-th/ 9501057]

7. N. Maggiore, Int. J. Mod. Phys. A 10, 3937 (1995). [hep-th/ 9412092]

8. N. Maggiore, O. Piguet, S. Wolf, Nucl. Phys. B 458, 403 (1996) [Erratum-ibid. B 469, 513 (1996)] [hep-th/9507045]

9. N. Maggiore, O. Piguet, S. Wolf, Nucl. Phys. B 476, 329 (1996). [hep-th/9604002]

10. K. Ulker, Mod. Phys. Lett. A 17, 739 (2002). [hep-th/0108062]

11. A. Blasi, O. Piguet, S.P. Sorella, Nucl. Phys. B 356, 154 (1991)

12. W. Hollik, E. Kraus, D. Stockinger, Eur. Phys. J. C 23, 735 (2002). [hep-ph/0007134]

13. W. Hollik, E. Kraus, M. Roth, C. Rupp, K. Sibold, D. Stockinger, Nucl. Phys. B 639, 3 (2002). [hep-ph/0204350]

14. M. Golterman, Y. Shamir, Phys. Rev. D 82, 105003 (2010). [arXiv: 1004.3860 [hep-th]]

15. W. Siegel, Phys. Lett. B 84, 193 (1979)

16. L.V. Avdeev, G.A. Chochia, A.A. Vladimirov, Phys. Lett. B $\mathbf{1 0 5}$ $272(1981)$

17. D. Stöckinger, JHEP 0503, 076 (2005). [arXiv:hep-ph/0503129]

18. L.V. Avdeev, A.A. Vladimirov, Nucl. Phys. B 219, 262 (1983)

19. R.V. Harlander, D.R.T. Jones, P. Kant, L. Mihaila, M. Steinhauser, JHEP 0612, 024 (2006). [arXiv:hep-ph/0610206]

20. I. Jack, D.R.T. Jones, P. Kant, L. Mihaila, JHEP 0709, 058 (2007). [arXiv:0707.3055 [hep-th]]

21. J.C. Collins, Nucl. Phys. B 80, 341 (1974)

22. J.C. Collins, Nucl. Phys. B 92, 477 (1975)

23. P. Nogueira, J. Comput. Phys. 105, 279 (1993)

24. R. Harlander, T. Seidensticker, M. Steinhauser, Phys. Lett. B 426 , 125 (1998). [hep-ph/9712228]

25. T. Seidensticker. [hep-ph/9905298]

26. J.A.M. Vermaseren. arXiv:math-ph/0010025

27. S.A. Larin, F.V. Tkachov, J.A.M. Vermaseren (1991) preprint NIKHEF-H-91-18

28. L.V. Avdeev, O.V. Tarasov, Phys. Lett. B 112, 356 (1982)

29. I. Jack, D.R.T. Jones, C.G. North, Phys. Lett. B 386, 138 (1996). [arXiv:hep-ph/9606323] 